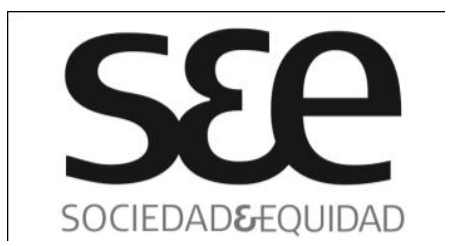

\title{
Arroz con leche ¿Me quiero casar?
}

\author{
Arroz con leche ¿l want to marry?
}

$\begin{array}{ll}\text { Nombre: } & \text { Toli Hernández Morales }\left({ }^{1}\right) \\ \text { Filiación: } & \text { Colectiva Ideas Sin Género } \\ \text { País: } & \text { Chile } \\ \text { Correo: } & \text { anatolia7@yahoo.com }\end{array}$

\section{RESUMEN}

El objetivo de este texto es reflexionar sobre algunos aspectos del sujeto y el tipo de ciudadanía que habita el debate sobre matrimonio homosexual desarrollado en Chile. Para ello, revisa algunas tensiones entre los enfoques de ciudadanía liberal y pluralista, observables en las posturas del movimiento de lesbianas, trans, gays, bisexuales, intersex o movimiento LTGBI. De esta manera, problematiza la relación entre ciudadanía, movimiento LTGBI y matrimonio relevando algunos dilemas respecto de la concreción de un enfoque pluralista. Dichos dilemas -plantea el texto- se basan en el no cuestionamiento de la heterosexualidad y en la ausencia de un enfoque interseccional que articule el análisis conjunto de distintas variables de exclusión. Lo indicado, se ve favorecido por la reproducción de una razón hegemónica que apoya la reducción de la noción de ciudadanía a un estatus legal. Es por esto, que se enfatiza el análisis de la heterosexualidad como una institución y no como una mera práctica sexual y la inclusión del enfoque interseccional para amplificar la libertad del Otra/a. Lo planteado es presentado, al finalizar, como una apuesta a la radicalización del pluralismo en la construcción de ciudadanía.

\section{ABSTRACT}

This essay seeks to reflect on some aspects of the subject and the kind of citizenship that inhabits the homosexual marriage debate that has taken place in Chile. Thus, it reviews some tensions between the liberal citizenship approach and the pluralistic approach, visible in the standpoints of lesbians, trans, gays, bisexuals and

\footnotetext{
${ }^{1}$ La autora es activista lesbofeminista. Profesora en Educación Básica y Magíster en Evaluación y Currículum por la Universidad de Santiago de Chile. Actualmente cursa el Doctorado en Estudios Latinoamericanos de la Universidad de Chile. Sus líneas de investigación se centran en ciudadanía, derechos y expresiones hegemónicas en el movimiento de diversidad sexual.
} 


\section{S\&e \\ Sresenow}

intersex people or the so called LTGBI movement. The text problematizes the relationship between citizenship, the LTGBI movement and marriage highlighting some dilemmas regarding the possibility of a pluralistic approach. It is argued that these dilemmas relate to the fact that heterosexuality is not questioned and the absence of an intersectional approach that articulates an analysis of different factors of exclusion. This is favored by the reproduction of a hegemonic reason that supports the reduction of the notion of citizenship to a legal status. This is why it emphasizes the analysis of heterosexuality as an institution and not just as a sexual practice and the inclusion of an intersectional approach to amplify the freedom of the Other's. Finally, the radicalization of pluralism in citizenship building is promoted.

PALABRAS CLAVES: Pluralismo, ciudadanía LTGB, matrimonio.

KEYWORDS: Pluralism, LTGB citizenship, marriage

\section{1.- De ¿razón? Ciudadanía y matrimonio}

El enfoque liberal de ciudadanía refiere a la propuesta clásica de Marshall centrada en la posesión de derechos civiles, políticos y sociales. Esta es cuestionada, entre otros, por la universalidad del sujeto que promociona, la no incorporación de la diversidad creciente de las sociedades modernas, la preservación de espacios de exclusión de la democracia representativa. El pluralismo reacciona frente a estos dilemas liberales e intenta establecer múltiples sentidos que afirmen que los grupos independientemente de sus diferencias- gocen del mismo estatus social. Una afirmación general -basada en una mirada latinoamericana- es que el movimiento LTGBI (Lesbianas, Transexuales, Gays, Bisexuales e Intersexuales) intenta descentrar la universalidad del sujeto provisto por la hegemonía cultural. Esto, para pluralizar la realidad a través de la reformulación de la diferencia como derecho y del reconocimiento de la libertad e igualdad de todas las personas.

En este punto es pertinente la definición de derechos sociales de Marshall, en donde algunos inscriben al matrimonio: "El elemento social abarca todo el espectro, desde el derecho a la seguridad y a un mínimo bienestar económico al de compartir plenamente la herencia social y vivir la vida de un ser civilizado conforme a estándares predominantes en la sociedad" (Marshall, 1949: 302). El matrimonio corresponde a un estándar predominante. Así es como el movimiento LTGBI lo acoge, sintetizando su ideario de igualdad. El liberal John Rawls plantea que la estructura básica de la sociedad se regula por "una concepción política de la justicia que es el foco de un consenso traslapado de doctrinas comprensivas razonables" (Rawls, 1996: 63). Lo anterior nuevamente nos lleva al predominio Marshalliano de cómo se configura lo razonable de Rawls y cuál es la posición de lo que no es asumido como 


\section{SEe}

tal. La razón liberal rawlsiana excluye lo irrazonable. Se configura así un conflicto entre liberalismo y pluralismo, pues si bien el primero lo admite, no ha podido implementarlo al naturalizar la selección de una "razón" que afirma a unos y oculta a otras.

Un ejercicio ayuda a esclarecer lo anterior:

Imagine a una pareja homosexual que adhiere al Acuerdo de Vida en Pareja - AVP ${ }^{3}$. ¿Cómo es? ¿Qué hace? ¿En dónde está? ¿Qué ropa usa?

¿Cómo imaginaron a quienes formaban esa pareja? ¿Eran pobres? ¿Eran indígenas? ¿Tenían $\mathrm{VIH}$ ? ¿Eran migrantes? ${ }^{4}$

Las respuestas a estas preguntas se vinculan a la selección cultural; la selección de la razón. La razón corazón del sistema de creencias de la corriente liberal de ciudadanía, gesta que cuando se nombra a un homosexual -para este caso una pareja homosexual- la imagen seleccionada culturalmente sea la de una que es occidental, blanca, burgués, clase media. Dicha universalización que responde a idearios hegemónicos dominantes excluye al pobre, indígena, negro y también a la travesti, al maricón, a la tortillera.

Con base en lo anterior, se reilumina el carácter cultural de la ciudadanía a través del condicionamiento impreso en la demanda por matrimonio del movimiento de lesbianas, trans, gays, bisexuales, intersex o movimiento LTGBI coherente con los idearios hegemónicos ${ }^{5}$. Para Rubin (Vance, 1989: 131) toda conducta erótica es mala a menos que exista una razón que la salve. Visto así, la homosexualidad a través del

\footnotetext{
${ }^{3}$ El AVP es la fórmula propuesta por políticos de derecha para proteger derechos de salud, previsionales, de herencia de las parejas del mismo sexo, restringidos solo a parejas formadas por personas de distinto sexo que han firmado pacto matrimonial.

${ }^{4}$ Incluí, con algunas modificaciones, este mismo ejercicio en el texto "Lesbianas y Acuerdo de Vida en pareja" publicado en la página de la Revista Pluma y Pincel: http://www.plumaypincel.cl/index.php?option=com_content\&view=article\&tid=678:lesbianas y-acuerdo-de-vida-en-pareja-avp-isig\&catid=39:debate

${ }^{5}$ Para efectos de este trabajo vale precisar que el movimiento lésbico y movimiento trans (travestis, transexuales y transgéneras) no son lo mismo que movimiento de lesbianas, trans, gays, bisexuales, intersex o LTGBI. Existen enunciaciones políticas que aluden a un particular y específico movimiento lésbico y trans conformado exclusivamente por lesbianas o personas trans. Dichas articulaciones informan sobre diferenciaciones políticas entre estos. Pese a la creencia de que el movimiento LTGBI (Lesbianas, Trans, Gays, Bisexuales) engloba al conjunto de estas voces, la historia acusa conflictos plenamente vigentes basados en la gobernabilidad gay dominante, en la subrepresentación de las voces lésbicas y trans, entre otras, que han determinado un fenómeno permanente de escisión de lesbianas y trans. Cabe indicar que el movimiento bisexual e intersex posee escasa visibilidad en toda Latinoamérica.
} 


\section{SEe}

matrimonio obtiene una razón que valida su existencia, pues es razonable desear acceder a él y con ello ascender en el sistema jerárquico de la sexualidad, en cuya cima -describe Rubin- se ubican las parejas heterosexuales matrimoniadas.

Lo anterior tensiona el sentido pluralista LTGBI, ya que dicho ascenso refuerza el modelo normativo dominante de la sexualidad. Lo indicado dirige la atención a una ciudadanía reducida a un estatus legal que inhibe el desarrollo de condiciones favorecedoras de procesos más participativos que diversifiquen el bien común. Si bien el movimiento LTGBI pluraliza el ideario universal del sujeto al exterior, tensiona dicha pluralización -al interior y también al exterior- al universalizar la demanda matrimonial que devuelve a su sujeto a la noción convencional y hegemónica del mismo.

\section{2.- Dilemas de la demanda matrimonial LTGBI}

La demanda matrimonial del movimiento LTGBI implica el acceso a privilegios sociales y económicos vedados. Sin embargo -esto que es justo- favorece la exclusión de aquellas/os que no deseen casarse, pese a que se ha insistido en que, de ser promulgada una ley al respecto, será de decisión libre el acceder o no a ella. Esta libertad no es tal, pues al formularse la normatividad del matrimonio, se naturaliza la obligación de adquirirlo, ya que es afirmado como única vía que resuelve conflictos de filiación, patrimonio, seguro social de las parejas homosexuales, es decir, beneficios a los que se debiera acceder sin restricción son restringidos a esta figura. Al movilizar la superación de los problemas a través de él, se le refuerza parafraseando a Preciado- como condición de acceso a la ciudadanía. Lo anterior sigo con Preciado- enmascara la violencia propia de las estructuras conyugales y familiares (Carrillo, 2007: 246).

El matrimonio se transforma así en un bien común, en un prerrequisito universal de los derechos. Volviendo a Marshall, se refuerza culturalmente como estándar predominante de los contenidos que definen el acceso a posiciones de igualdad y libertad. Lo indicado expone un racionalismo político que transforma al matrimonio -siguiendo a Butler (2001)- en un espacio de legitimación que ilegitima a su vez lo irrepresentable, pues irrepresentable es una pareja gay fuera de este código normativo. De este modo, la demanda matrimonial opera como instrumento de homonormatividad, es decir, "como un constructo cultural que convierte a la homosexualidad en un espacio normativizado de disidencia sexual" (Moreno, Pichardo, 2006:151). Siguiendo esta lógica, el matrimonio refuerza la selección cultural propiciada por el sistema sexo/género, pues convierte al sexo (hombre/mujer) y al género (femenino/masculino) en una frontera a la hora de establecer prácticas e identidades. Esto último permite relevar la tensión de este debate, que no se centra en la legitimidad de la demanda matrimonial sino en su rol 


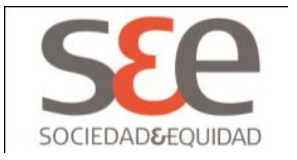

de agente normativo que se subordina a lo razonable, que es pensarse en los patrones de estabilidad, fidelidad, protección patrimonial que otorga el concepto de familia heterosexual. Este tránsito, que no promociona nuevas formas legales que vinculen a las personas, que no asume otras formas de relacionamiento afectivo, que tampoco aporta nuevas formas de relación entre sociedad y Estado, reproduce la heterosexualidad y transforma a la homosexualidad en su aparejo normativo.

El debate en torno al matrimonio en Chile ha sido levantado por algunos sectores del movimiento LTGBI que se posicionan como voces hegemónicas dominantes. Estas han organizado el ideario democrático de la diversidad sexual aunque no sean del todo coherentes con las necesidades manifestadas por la comunidad. En el 2007, en la investigación publicada como "Política, Derechos, Violencia y Diversidad Sexual: Primera Encuesta Marcha por la Diversidad Sexual", solo el $3.95 \%$ de las 486 personas que aplicaron consideró como demanda política LTGB al matrimonio. En ese momento la ley antidiscriminatoria ocupaba el $33.5 \%$ (Barrientos et. al, 2008: 52). En el 2010 existió una convocatoria a lesbianas activistas para incidir al respecto que no prosperó. En el 2011, pese a que filiación y parentesco fueron parte de la sistematización de demandas lésbicas elaborada para la mesa de trabajo lésbica metropolitana, ésta focalizó sus esfuerzos en la salud integral (Hernández, 2011:6). A lo menos, hasta el 2008, activistas travestis con las que la autora de este texto desarrolló un estudio sobre vulnerabilidad, sostenían discursos del tipo: "iQue me importa que se casen los colas si a nosotras nos siguen matando en la calle!" Lo indicado, alerta sobre mecanismos de poder que transforman la demanda matrimonial de un grupo en la demanda que se posiciona públicamente como la de todas. Esto entronca con lo indicado ya sobre selección cultural.

El eslogan "Ni igualadas, ni aparecidas. Ni con leyes chantas seremos convencidas" que se tomó el inicio de la Marcha 2011 convocada por organizaciones LTGBI, informa sobre unas realidades periféricas resistentes a la dominancia cultural asociada al Acuerdo de Vida en Pareja -AVP-. Estas, entre otras, denuncian el uso del AVP por la derecha como parte de una estrategia dirigida a infiltrar los movimientos sociales, la debilitación de las demandas de las minorías sexuales y el asentamiento de un ideario de transversalidad que responde a necesidades económicas de organizaciones LTGBI supeditadas a los ordenamientos de la derecha política. Así, también informan sobre la implementación de una agenda política LTGBI igualizante, normalizadora e higiénica de la homosexualidad en Chile.

Lo expresado, despierta la alerta sobre la confrontación liberalismo/pluralismo al interior del movimiento LTGBI. El proceso histórico de su constitución en LAC desde la construcción de una identidad unitaria en una primera etapa a partir de los 70 (Bellucci, Rapisardi, 2006: 49; Figari, 2009: 189) a la constitución del movimiento LTGBI en la década de los '90, informa sobre tensiones 


\section{SEe}

identitarias que generaron divisiones visibilizando que la ciudadanía homosexual no posee un sexo neutro, ni tampoco una raza o una clase que lo sea (Mogrovejo, 2004:86). Se informa de esta manera sobre la existencia de relaciones conflictivas entre "masculinidad y feminidad homosexual" que exacerban diferencias. Estas reproducen el ejercicio convencional de ciudadanía en donde lo público -el espacio en donde se construye la política- es ejercido por una homosexualidad masculina hegemónica, es decir, por aquella en donde -como dice el activista Víctor Hugo Robles en el periódico The Clinic-, "Las feas, las sidosas, las pobres, no son bienvenidas" . Cabe indicar en este punto que la gobernabilidad LTGBI se caracteriza por un liderazgo gay en donde la enunciación de otras identidades como las LTBI están en lo absoluto subrepresentadas ${ }^{7}$.

En síntesis: el sentido pluralista del movimiento LTGBI se tensa en un sujeto que tiende a universalizarse, en el reduccionismo de sus demandas políticas y en la hegemonía dominante que opera en su interior y que le otorga un conflicto de representatividad. Así es como muchas veces reproduce el sexismo, el racismo, el clasismo. Desde esta perspectiva no queda claro qué introduce analíticamente la categoría de sexualidad a una nueva concepción de ciudadanía, lo que nuevamente entronca con la reproducción de la práctica cultural dominante.

\section{Arroz con leche... ¿Me quiero casar?}

La tendencia universalista del sujeto -afincada en el estándar predominante de Marshall, en la razón de Rawls, en el propio liberalismo- implanta idearios de bien común que refuerzan la reproducción de la cultura dominante. El matrimonio -al menos así como ha sido presentado, asociado a un sujeto gay hegemónico- abre espacios en la heterosexualidad a costa de su refuerzo como bien social universal. Así, fortalece la carga social simbólica del matrimonio expandiendo sus redes normalizadoras que van más allá del tipo de prácticas sexuales que realicen quienes lo contraigan. En esta escena, radicalizar el pluralismo de la ciudadanía asume principios de libertad e igualdad para todas. Dichos principios invocan la denuncia del carácter institucional de la heterosexualidad realizada por el feminismo radical y los aportes del feminismo negro sintetizados en la aplicación de un enfoque de interseccionalidad que entrecruce distintas variables de discriminación reproducidas en las demandas y prácticas LTGBI.

\footnotetext{
${ }^{6}$ Activista gay chileno conocido como El che de los gays; reconocido públicamente como un disidente de las políticas movilizadas por organizaciones LTGB hegemónicas.

${ }^{7}$ Revisar Héctor Salinas Hernández "Políticas públicas de disidencia sexual: apuntes para una agenda". En Disidencia sexual e identidades sexuales y genéricas (2006) México, CONAPRED. El autor plantea que la gobernabilidad se refiere a la forma de hacer gobierno en el espacio público; allí se pone en juego el carácter pluralista de la democracia y de la ciudadanía.
} 


\section{SEe}

En virtud de lo indicado y de dotar -aunque es majadero decirlo- a esta lucha de posiciones que amplíen la posibilidad de reordenar, deconstruir, desobedecer, se introducen algunos elementos considerados de importancia:

La heterosexualidad es una institución y no una mera práctica sexual. Actúa como mecanismo de orden que normaliza el comportamiento de toda la sociedad; trasciende las voluntades individuales al identificarse con la imposición de una aspiración dominante que se naturaliza hasta transformarla en un bien social universal. Su funcionamiento se basa en la elaboración de normas poco flexibles que constituyen su carácter opresivo. El "pensamiento hétero" universaliza conceptos, formula leyes generales que valen para todas las sociedades, épocas, individuas/os creando categorías que "no tienen sentido más que en la heterosexualidad o en un pensamiento que produce la diferencia de los sexos como dogma filosófico y político" (Wittig, 1979: 52). O sea, la institución hétero oprime a todas/todos, por lo que se debe dilucidar qué se pone en juego al pensar en la libertad e igualdad. Este señalamiento promueve -más allá de posturas en contra o a favor del matrimonio- la construcción de un lenguaje propio no subordinado a la heterosexualidad. Para que la sexualidad LTGB transforme los contenidos de la ciudadanía, ésta debe cuestionar el carácter de heterosexual de todo.

Enfoque interseccional. La libertad se amplifica en la liberación del Otro/a. Es necesario abordar las exclusiones específicas, pero asumidas en relación a la reproducción de la discriminación que signifiquen, de tal modo que se respete que “a) los derechos de las minorías no deben permitir que un grupo oprima a otros grupos; y b) tampoco deben permitir que un grupo oprima a sus propios miembros" (Kymlicka, 1996: 266). Se trata de evitar que el reconocimiento de la diferencia genérica sexual apoye la exclusión de otras diferencias. Es en este contexto en donde el aporte de la interseccionalidad es significativo, pues apostar a la intersección de las categorías posibilita la visibilidad de unas que se ocultan en discursos que tienden a seleccionar al dominante" (Lugones, 2003: 63) La mirada interseccional propicia construcciones pluralistas que articulan el análisis conjunto de diferencias que constituyen desigualdad -sexo, género, raza, clase- para desestabilizar sus impactos múltiples en los sujetos.

Finalmente, este texto implícitamente promociona hurgar en lo oculto, en lo que domestica los deseos y somete a las sujetos a los principios de la ley. Interroga los costos del dominio de la institucionalidad heterosexual. Es pensamiento básico de resistencia que problematiza la ciudadanía sustantiva -basada en derechos- y la cuestiona, interroga, interpela. De este modo, se intenta aportar a que el maricón no sea negado como categoría política, porque le pega a las mujeres; a que se deseche el ropaje comercial que el mercado le infringió a la homosexualidad; a que no se oculte la violencia que subyace al amor, pues por amor se mata y también se 


\section{SEe}

adquiere el $\mathrm{VIH}^{8}$. Se trata de apoyar que las masculinidades no se mantengan iguales basándose para ello en la desobediencia crítica de todos los tipos: de la cultura, de sus conceptos, de las leyes, del género, del mercado. De todo.

\section{Referencias Bibliográficas}

Carrillo, Jesús (2004). Entrevista con Beatriz Preciado (pp. 245-261) Recuperado de <http://www.interfaceg2g.org/midia/g2g/preciado.pdf>

Barrientos, Jaime (et. al) (2008). Política, Derechos, Violencia y Diversidad Sexual: Primera encuesta Marcha por la diversidad sexual. Colección documentos. CLAM. Río de Janeiro.

Butler, Judith (2001). La cuestión de la transformación social. En Berk Gerssheim, E. y otras (2001), Mujeres y transformaciones sociales (pp.7-30). Barcelona: Ed. El Roure.

Hernández, Iris (2011). Borrador, documento en construcción: Derechos y Exigencias políticas Lésbicas y Bisexuales. Trabajo realizado para la mesa lésbica metropolitana.

Kymlicka, Will. (1996). Ciudadanía Multicultural. Una teoría Liberal a los derechos de la Ciudadanía. Barcelona: Ed. Paidós.

Marshall, T y Bottomore T.(1997). Ciudadanía y clase social. Madrid, Alianza Editorial.

Mogrovejo, Norma (2004). Relevancia de las lesbianas en América Latina: La recuperación de nuestra historia. En Drucker Peter (coord.) Arcos Iris Diferentes (pp.86-89).México DF, Siglo XXI editores.

\footnotetext{
${ }^{8}$ Se alude, en primer lugar, a la campaña chilena realizada por el Servicio Nacional de la Mujer en donde el eslogan principal es: "Maricón es el que le pega a una mujer". Lo citado en segundo lugar, se relaciona con las series norteamericanas que articulan un ideario de riqueza y sofisticación LTGB muy consumido, vale decir, por esta comunidad. El tercer punto alude a la no problematización del amor LTGBI desde las violencias que lo habitan.
} 


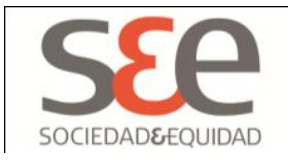

Moreno Ángel y Pichardo José Ignacio (2006). “Homonormatividad y existencia sexual. Amistades peligrosas entre género y sexualidad" (pp. 143-156). Revista de Antropología Iberoamericana. Enero-febrero volumen 001. Antropólogos Iberoamericanos en Red. Madrid- España.

Rawls, John (1993). Liberalismo Político. México: Fondo de Cultura Económica.

Rubin, Gayle (1989). Notas para una teoría radical de la sexualidad. En: Vance, Carole S. (Comp.) Placer y peligro. Explorando la sexualidad femenina (pp. 113-190). Madrid: Ed. Revolución.

Salinas, Héctor (2007). Políticas públicas de disidencia sexual: apuntes para una agenda. En Consejo Nacional para la Prevención de la Discriminación - CONAPRED Disidencia sexual e identidades sexuales y genéricas (pp. 21 -29). México DF.

Wittig, Monique (2006). El pensamiento heterosexual. En El pensamiento Heterosexual y otros ensayos (pp. 45-57) Barcelona, Editorial Egales.

\section{Periódicos}

The Clinic: "El che de los gays contra AVP e IGUALES: "No somos bienvenidas, los maricones, feos, pobres, afeminados y sidosas” 06/10/2011. Ed. № 414, Santiago de Chile. 